\title{
Neighbor Discovery based on Adaptive Hello Messaging Scheme On-Demand MANET Routing Protocols using PSO
}

\author{
Sukhvinder Kaur \\ M.Tech. Research Student, \\ Department of Computer Science and \\ Engineering ,Sri Guru Granth Sahib World \\ University (SGGSWU)Fatehgarh Sahib.
}

\author{
Sheenam Malhotra \\ Assistant professor \\ Department of computer science and \\ Engineering,Sri Guru Granth Sahib World \\ University (SGGSWU)Fatehgarh Sahib.
}

\begin{abstract}
Mobile ad-hoc network is an infrastructure less wireless adhoc network in which mobile nodes communicate with each other through wireless links without the need of any centralized administration. Overhead goes high due to neighbor discovery messages in the MANET routing protocols, such as in AODV, DYMO. This paper implements both the protocols adaptive and then for optimization PSO is applied. Simulation results show that the proposed scheme reduces energy consumption and suppressing unnecessary hello messaging.
\end{abstract}

\section{Keywords}

AODV, DYMO, Ad- hoc Routing, Hello messaging, Network Overhead, Energy Saving.

\section{INTRODUCTION}

A Mobile Ad hoc Network (MANET) is a collection of mobile nodes (MNs) that cooperatively communicate with each other without any pre-established infrastructures such as a centralized access point [2]. Wireless networks can be classified as: Infrastructure based network, Infrastructure less network. In infrastructure based wireless network nodes communicate to the Internet by using access points or base stations. In infrastructure less wireless networks nodes communicate with each other through wireless links without the need of access points. For example transferring of data between two mobiles using Bluetooth is an infrastructure less or ad-hoc network. Due to dynamic topology of MANETs some problems occurs like availability of nodes, power and battery lifetime of each node. Some common applications of MANETs are military operations, disaster recovery, wireless sensor network, and remote geographical area where no access points or base stations used for communication [5]. Routing Protocols can be classified into three categories as shown in the fig. 1: Reactive (On-demand) Routing Protocols, Proactive (Table-driven) Routing Protocols and Hybrid Routing Protocols.
MANET routing protocols are:

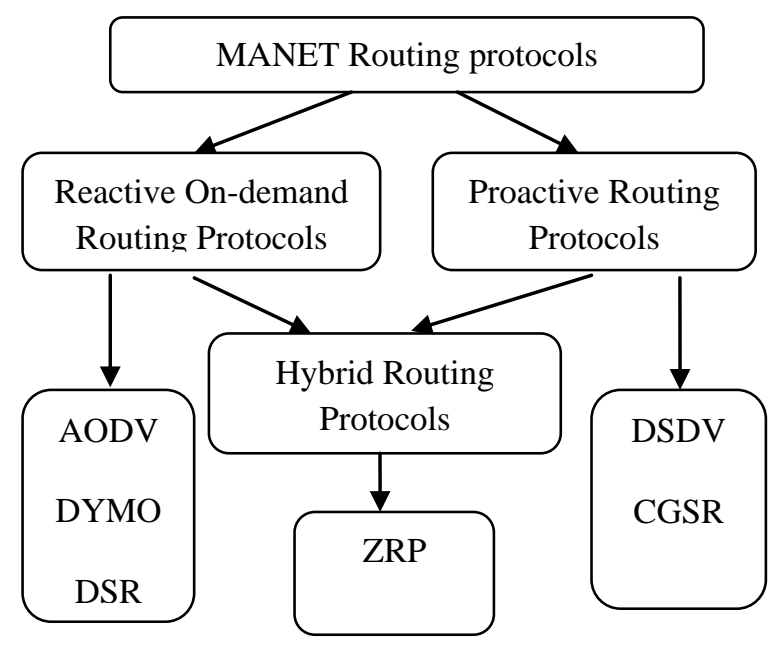

Fig.1 MANET Routing Protocols

\section{MANET ROUTING PROTOCOLS}

Reactive (On-Demand) Protocols

Reactive routing protocols are also called On-demand routing protocols because route is only constructed when nodes want communication with each other. If any node wants to send data to other node then reactive routing protocol start searching route in an on-demand manner and construct a connection for sending and receiving data [3]. Some common examples of reactive routing protocols are the following: AODV (Ad-hoc on-demand routing protocol), DYMO (Dynamic MANET On-demand protocol), and DSR (Dynamic Source Routing). The Ad-hoc On-Demand Distance Vector (AODV) routing protocol is designed for use in ad-hoc mobile networks. AODV is a reactive protocol: the routes are created only when they are needed [19]. AODV determines a route to a destination only when a node wants to send a packet to that destination. A source node that wants to send a message to a destination for which it does not have a route, broadcasts a request RREQ packet. Request (RREQ), Route Reply (RREP), Route Error (RERR) is the control messages used to find a path from source to destination. AODV consists of two protocol operations: Route discovery and Route maintenance. The Dynamic MANET On-demand (DYMO) routing protocol is a newly proposed protocol currently defined in an IETF Internet-Draft in its sixth revision and is still work in progress. It operates similarly to AODV. DYMO does not add extra features or extend the AODV protocol, but rather simplifies it, while retaining the basic mode of operation [3]. DYMO is also consisting of two 
protocol operation: Route discovery and Route maintenance. Dynamic source routing (DSR) is based on source routing where the source specifies the complete path to the destination in the packet header [3]. This Protocol is composed of two essential parts of Route discovery and Route maintenance [4].

\section{Proactive Routing Protocols}

Proactive routing protocols are also known as table driven protocol. In these protocols, each node maintains a routing table consisting of routing information to every other node in the network [4]. Examples of proactive routing protocols are following: DSDV, CGSR etc. Dynamic DestinationSequenced Distance-Vector Routing Protocol (DSDV) is developed on the basis of Bellman-Ford routing algorithm with some modifications [19]. It is suitable for creating ad-hoc networks with small number of nodes. DSDV is not suitable for highly dynamic networks. Cluster Gateway Switch Routing Protocol (CGSR) considers a clustered mobile wireless network instead of a 'flat' network. One drawback of this protocol is that, frequent change or selection of cluster heads might be resource hungry and it might affect the routing performance [19].

\section{Hybrid Routing Protocol}

Hybrid protocols combine the features of reactive and proactive protocols. The main advantage of hybrid routing protocol is that the proactive routing used for small distance and reactive routing used for long distance [5]. There are various popular hybrid routing protocols for MANET like ZRP. ZRP is suitable for wide variety of MANETs, especially for the networks with large span and diverse mobility patterns. ZRP uses a query control mechanism to reduce route query traffic by directing query messages outward from the query source and away from covered routing zones [19].

\section{Particle Swarm Optimization Algorithm (PSOA)}

PSO is an evolutionary computation technique like genetic algorithms. Since PSO have many advantages such as comparative simplicity, rapid convergence and little parameters to be adjusted, it has been used in many fields such as mechanical, chemical, civil, aerospace design etc [15].The process, in which a bacterium moves by taking small steps while searching for nutrients, is called chemo taxis and key idea of PSOA is mimicking chemo tactic movement of virtual bacteria in the problem search space, individual bacterium communicate to other by sending signals. This technique is also inspired by the social foraging behavior like ant colony and bacterial foraging optimization algorithm. It attracts the researchers due to its efficiency in solving real world optimization problems and gives better results than traditional methods of problems solving.

\section{PROPOSED WORK}

This section firstly presents the network model and then formulates the adaptive scheme for hello messaging. Node mobility follows a model, there is several mobility models used in the evaluation of MANET protocols. The exponential and pareto-optimal model is one the most commonly used mobility models for simulations of MANETs. In this model, each node selects a random destination, uniformly distributed within the two-dimensional space. In conventional hello messaging scheme before a packet is sent, status of neighbor nodes should be recognized first so as to recognize if there is a link failure with one of its neighboring nodes. After the outputs of AODV, AODV-AH, DYMO, and DYMO-AH are taken then we apply PSO and compare the output of all. This technique gives better results than traditional methods of problems solving.

\section{IMPLEMENTATION WORK}

Mobility model chosen is exponential and pareto-optimal model, in this model node chooses a random destination point. In the simulation we concentrated on two performance metrics: network overhead and energy consumption.

Table 1 Simulation Parameters

\begin{tabular}{|c|c|}
\hline Parameters & Values \\
\hline Simulator & MATLAB \\
\hline Number of Nodes & O,40,60 \\
\hline Pause Time & $\begin{array}{c}\text { Exponential and } \\
\text { Pareto-optimal }\end{array}$ \\
\hline Mobility Model & 10mbs \\
\hline Data Rate & $512 \mathrm{~kb}$ \\
\hline Packet Size & AODV, DYMO \\
\hline Protocols &
\end{tabular}

Fig. 2 shows the comparison of energy consumption between DYMO and DYMO-AH. Each node is assigned with 1 joule of energy initially. As time goes on increasing DYMO consumes energy quickly due to periodic hello messaging scheme and hence more energy consumption and battery drain will take place. When DYMO-AH energy consumed is less due to less transmissions and reception of hello messages. The $\mathrm{X}$-axis represents number of flows and $\mathrm{Y}$-axis represents consumed energy (joules).

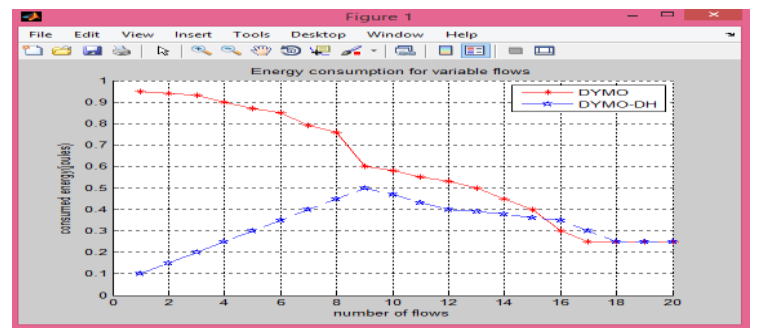

Fig.2 Energy consumption for variable flows

Fig. 3 shows the hello packet overhead. Here number of the hello packets is decreased by as much as half due to the proposed scheme, because as number of nodes will increase, the number of hello packets received by a node will also increase. The $\mathrm{X}$-axis represents no. of nodes and $\mathrm{Y}$-axis represents hello packet ratio.

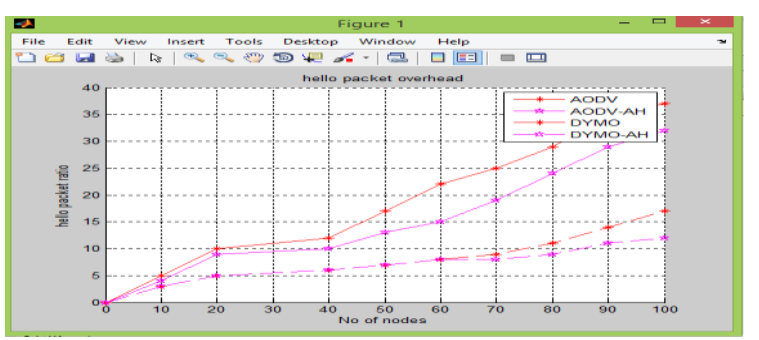

Fig. 3 Hello packet overhead

Fig. 4 compares the energy consumed by AODV and AODVAH. Initially each node has 160 joules of energy. As number of nodes will increase AODV consumes energy quickly, while AODV-AH due to the proposed scheme will reduce unnecessary hello messages hence, take energy consumption will take more time. The $\mathrm{X}$-axis represents no. of events occurred and $\mathrm{Y}$-axis represents energy consumed (joules). 


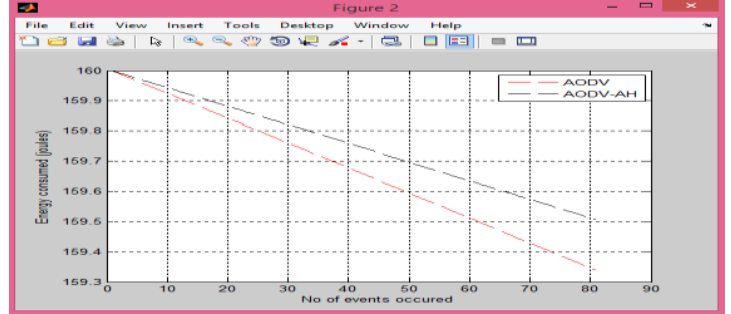

Figure 4 Energy consumption for AODV and AODV Adaptive

Fig.5 compares the energy consumed by DYMO and DYMO $\mathrm{AH}$. The graph shows the 160.05 joules energy, but initially each node has 160 joules of energy. As number of nodes will increase DYMO consumes energy quickly, while DYMO -AH due to the proposed scheme will reduce unnecessary hello messages hence, take energy consumption will take more time. The $\mathrm{X}$-axis represents no. of events occurred and $\mathrm{Y}$-axis represents energy consumed (joules).

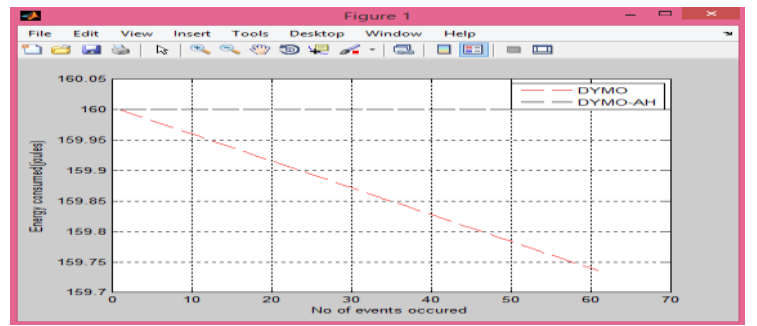

Fig. 5 Energy consumption for DYMO and DYMO Adaptive

Fig.6 shows the impact of PFD on the throughput high speed PFD uses a longer Hello interval than a low PFD. The X-axis represents max.speed $(\mathrm{m} / \mathrm{s})$ and $\mathrm{Y}$-axis aggregated throughput (mbps).

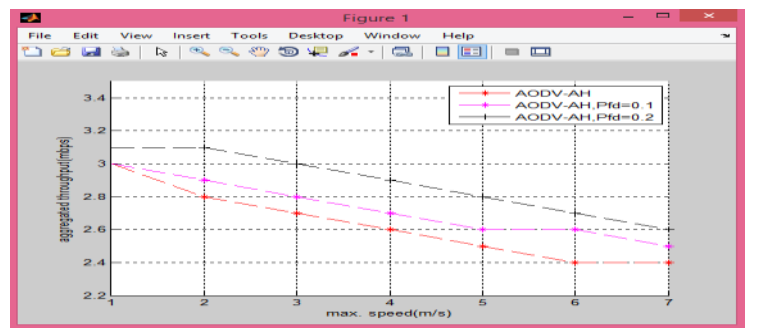

Fig. 6 Throughput for various max speed and PFD

Fig. 7 shows the particle swarm optimization (PSO) is applied in MATLAB. In this Fig. 7 shows the movement of particle after applying PSO technique.

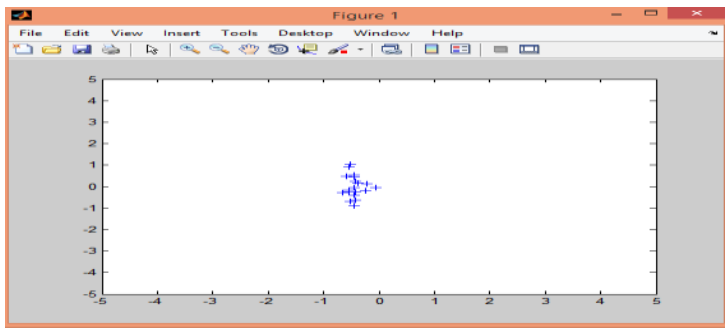

Fig. 7 Movement of particles

Fig.8 shows the output after PSO (Particle swarm optimization) is applied. On different number of nodes remaining energy of nodes is calculated. AODV-AHPSO results are much better than AODV and AODV-AH. The X- axis represents no. of nodes and $\mathrm{Y}$-axis represents remaining energy (joules).

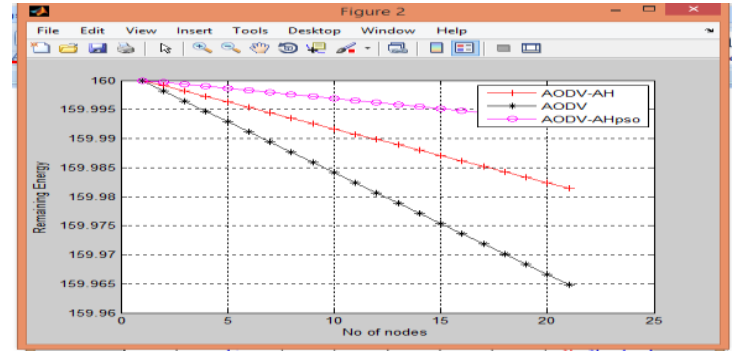

Fig. 8 Energy consumption with variable nodes of AODV, AODV Adaptive and AODV adaptive PSO

Fig. 9 shows the output after PSO is applied on DYMO. DYMO-AHPSO results are much better than DYMO and DYMO-AH. The $\mathrm{X}$-axis represents no. of nodes and $\mathrm{Y}$-axis represents remaining energy (joules).

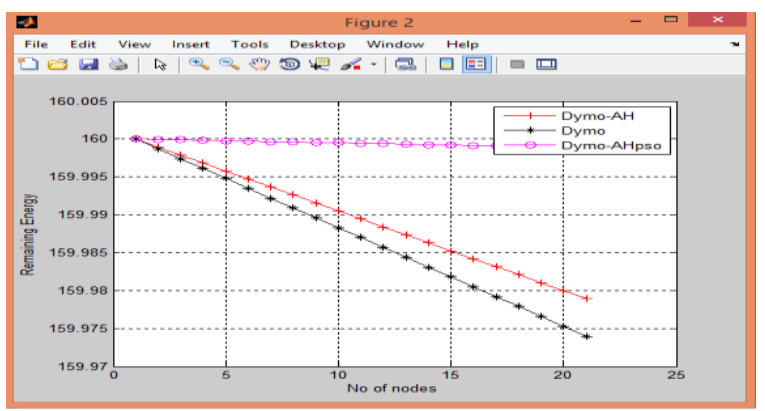

Fig. 9 Energy consumption with variable nodes of DYMO, DYMO adaptive and DYMO adaptive PSO

\section{CONCLUSION AND FUTURE SCOPE}

This paper evaluates the performance of MANET Routing Protocols i.e. Reactive Routing Protocols (AODV, DYMO). Adaptive hello messaging scheme is proposed to solve the problem related battery consumption and network overhead. Hello messaging scheme aims to suppress unnecessary hello messages while a neighbor discovery and also establish a reliable connection between the source nodes to the destination node. The future work suggests the development of modified version of the selected routing protocols which should consider different aspects of routing protocols such as rate of higher route establishment and faster speed of send the messages source to destination node. The future enhancement of existing technique is to implement more efficient routing protocols such as IPSO (Improved Particle Swarm Optimization)

\section{REFERENCES}

[1] Seon Yeong Han and Dongman Lee, "An Adaptive Hello Messaging Scheme for Neighbor Discovery in On-Demand MANET Routing Protocols" IEEE communications letters, Vol. 17, Page No.1040-1043, May 2013.

[2] Swati Dhawan,Vinod Saroha, Richa Dhawan, "Review on Performance Issues of Routing Protocols of Mobile Ad-hoc Networks", International Journal of Advanced Research in Computer Science and Software Engineering , ISSN: 2277 128X, Issue No. 6, Vol.3, Page No.10241029, June 2013 
[3] Anuj K.Gupta, Jatinder Kaur, Sandeep Kaur, "Comparison of DYMO,AODV,DSR and DSDV MANET Routing Protocols Over Varying Traffic", International Journal of Research in Engineering \& Applied Science (IJREAS), ISSN: 2294-3905,Issue No.2, Vol.1, Page No.71-83, October, 2011.

[4] Anit Kumar, Pardeep Mittal, "A Comparative Study of AODV \& DSR Routing Protocol in Mobile Ad-Hoc Networks", International Journal of Advanced Research in Computer Science and Software Engineering, ISSN: 2277 128X, Issue No. 5, Vol.3, Page No.658-663 May 2013.

[5] Jagdeep Kaur,Rupinder Kaur Gurm, "Performance Analysis of AODV and DYMO Routing Protocols in MANETs Using Cuckoo Search Optimization", International Journal of Advance Research in computer Science and Management Studies, ISSN: 2321-7782, Issue No.8, Vol.2, Page No. 236-247, August 2014.

[6] Jatinder Pal Singh, Anuj Kr. Gupta, "Performance Analysis of AODVv2 Protocol vs.AODV Protocol in MANET" International Journal of Emerging Technologies in Computational and Applied Sciences (IJETCAS), ISSN (print):2279-0047, ISSN (online):2279-0055, Page No.621-625, March-May

[7] Anuj K. Gupta, Harsh Sadawarti and Anil K. Verma, "Implementation of DYMO Routing Protocol", International Journal of Information Technology, Modeling and Computing (IJITMC), Issue No. 2, Vol.1, Page No.49-57, May 2013.

[8] Serene Bhaskaran, Ruchi Varma, Dr.Jayanta Ghosh, "A comparative study of GA, PSO and APSO: Feed point optimization of a patch antenna", International Journal of Scientific and Research Publications(IJSRP), ISSN 2250-3153 , Issue No. 5, Vol.3, Page No. 1-5, May 2013.

[9] Radha Thangaraj, Millie Pant, Ajith Abraham, and Vaclav Snasel, "Modified Particle Swarm Optimization With Time Varying Velocity Vector", International Journal of Innovative Computing, Information and Control (ICIC International), ISSN 1349-4198, Issue No.1(A), Vol.8, Page No.201-218, January 2012.

[10] M. R. AlRashidi, and M. E. El-Hawary, "A Survey of Particle Swarm Optimization Applications in Electric Power Systems", IEEE Trasaction on Evolutionary Computation,Page No.1-6, 2006.

[11] Sapna Katiyar, "A Comparative Study of Genetic Algorithm and the Particle Swarm Optimization"
AKGEC International Journal of Technology, Issue No.2, Vol.2, Page No.21-24.

[12] Prashant Kumar Maurya, Gaurav Sharma, Vaishali Sahu, Ashish Roberts, Mahendra Srivastava, "An Overview of AODV Routing Protocol", International Journal of Modern Engineering Research (IJMER), ISSN: 22496645, Issue No.3, Vol.2, Page No.728-732 May-June 2012.

[13] Manju, Ranjana Thalore, Jyoti, M.K Jha, "Performance Evaluation of Bellman-Ford, AODV, DSR and DYMO Protocols using QualNet in $1000 \mathrm{~m} \times 1000 \mathrm{~m}$ Terrain Area", International Journal of Soft Computing and Engineering (IJSCE), ISSN: 2231-2307, Issue No.6, Vol.2, Page No. 140-149, January 2013.

[14] Gunvir Kaur, Er. Sugandha Sharma, "Research Paper on Optimized Utilization of Resources Using PSO and Improved Particle Swarm Optimization (IPSO) Algorithms in Cloud Computing" International Journal of Advanced Research in Computer Science \& Technology (IJARCST), ISSN: 2347 - 8446 (Online), ISSN: 2347 - 9817 (Print), Issue No. 2, Vol.2, Page No. 499-595, April - June 2014.

[15] Vishal A. Rane, "Particle Swarm Optimization (PSO) Algorithm: Parameters Effect And Analysis", International Journal of Innovative Research and Development (ijird), ISSN: 2278 - 0211 (Online) ,Issue No.7,Vol.2, Page No. 8-16, July 2013.

[16] Sharvani G S, Cauvery N K, Dr.Rangaswamy.T, "Adaptie Routing Algorithm For Manet:Termite" International Journal of Next-Generation Networks (IJNGN),Issue No.1,Vol.1,Page No. 38-43, December 2009.

[17] Bharat Rathi, Dattatray V. Jadhav, "Network Intrusion Detection Using PSO Based on Adaptive Mutation and Genetic Algorithm" International Journal of Scientific \& Engineering Research, ISSN: 2229-5518, Issue No.8, Vol.5, Page No.142-144, August-2014.

[18] Anuj K. Gupta, Harsh Sadawarti, and Anil K. Verma, "MANET Routing Protocols Based on Ant Colony Optimization", International Journal of Modeling and Optimization, Issue No. 1,Vol. 2, Page No. 42-49, February 2012.

[19] Dr.S.S.Dhenakaran, A.Parvathavarthini, "An Overview of Routing Protocols in Mobile Ad-Hoc Network", International Journal of Advanced Research in Computer Science and Software Engineering, ISSN: 2277 128X, Issue No. 2, Vol. 3, Page No. 251-259, February 2013. 